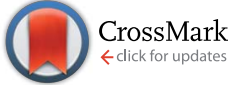

Cite this: J. Mater. Chem. A, 2015, 3 , 24071

Received 21st October 2015 Accepted 21st October 2015

DOI: $10.1039 / c 5 t a 08450 d$

www.rsc.org/MaterialsA

\section{Pushing efficiency limits for semitransparent perovskite solar cells $\uparrow$}

\author{
César Omar Ramírez Quiroz, ${ }^{\text {a }}$ levgen Levchuk, ${ }^{a}$ Carina Bronnbauer, ${ }^{\text {ab }}$ \\ Michael Salvador, ${ }^{\text {ac }}$ Karen Forberich, ${ }^{a}$ Thomas Heumüller, ${ }^{a}$ Yi Hou, $^{\text {ab }}$ \\ Peter Schweizer, ${ }^{d}$ Erdmann Spiecker ${ }^{d}$ and Christoph J. Brabec ${ }^{\text {ae }}$
}

\begin{abstract}
While perovskite-based semitransparent solar cells deliver competitive levels of transparency and efficiency to be envisioned for urban infrastructures, the complexity and sensitivity of their processing conditions remain challenging. Here, we introduce two robust protocols for the processing of sub-100 nm perovskite films, allowing fine-tuning of the active layer without compromising the crystallinity and quality of the semiconductor. Specifically, we demonstrate that a method based on solvent-induced crystallization with a rapid drying step affords perovskite solar cells with $37 \%$ average visible transmittance (AVT) and 7.8\% PCE. This process enhances crystallization with a preferential phase orientation presumably at the interface, yielding a high fill factor of $72.3 \%$. The second method is based on a solvent-solvent extraction protocol, enabling active layer films as thin as $40 \mathrm{~nm}$ and featuring room-temperature crystallization in an ambient environment on a few second time span. As a result, we demonstrate a maximum AVT of $46 \%$ with an efficiency of $3.6 \%$, which is the highest combination of efficiency and transparency for a full device stack to date. By combining the two methods presented here we cover a broad range of thicknesses vs. transparency values and confirm that solvent-induced crystallization represents a powerful processing strategy toward high-efficiency semitransparent solar cells. Optical simulations support our experimental findings and provide a global perspective of the opportunities and limitations of semitransparent perovskite photovoltaic devices.
\end{abstract}

\section{Introduction}

The development of innovative technologies with improved levels of sustainability that are capable to compete with today's state of the art photovoltaic devices is challenging, given their widely established deployment methods. Silicon technologies, nowadays leading the market, still have several advantages over second and third generation solar cells for conventional applications. One application with promising potential on getting into the fast track of becoming a realistic contender for silicon

${ }^{a}$ Institute of Materials for Electronics and Energy Technology (I-MEET), Department of Materials Science and Engineering, Friedrich-Alexander University Erlangen-Nuremberg, Martensstrasse 7, 91058 Erlangen, Germany. E-mail: omar. quiroz@fau.de

${ }^{b}$ Erlangen Graduate School in Advanced Optical Technologies (SAOT), Paul-GordanStr. 6, 91052 Erlangen, Germany

'Instituto de Telecomunicações, Instituto Superior Técnico, Av. Rovisco Pais, P-1049o01 Lisboa, Portugal

${ }^{d}$ Center for Nanoanalysis and Electron Microscopy (CENEM), Department Werkstoffwissenschaften, Friedrich-Alexander University Erlangen-Nuremberg, Cauerstraße 6, 91058 Erlangen, Germany

${ }^{e}$ Bavarian Center for Applied Energy Research (ZAE Bayern), Am Weichselgarten 7, 91058 Erlangen, Germany

$\uparrow$ Electronic supplementary information (ESI) available. See DOI: 10.1039/c5ta08450d technologies is semitransparent photovoltaics. The ability to implement semitransparent solar panels into consumer products such as building integrated elements, e.g. windows, or portable electronic devices relying on existing infrastructure could potentially lead to an economic boost in the field of photovoltaics. In this regard, organic photovoltaic (OPV) devices offer a unique potential given their characteristics including low manufacturing cost, lightweight, flexibility, intrinsic transparency and, most importantly, solution processability, desirable properties in "roll-to-roll" scalable protocols. Despite their strategic advantages, recent progress in up-and-coming new technologies such as perovskites has shown significant potential outshining OPV established technologies in terms of efficiency. The latest findings demonstrated efficiencies as high as $\mathbf{1 0 . 8 \%}$ for single-junction opaque OPV devices. ${ }^{\mathbf{1} 2}$ In contrast, perovskite photovoltaic devices have shown certified efficiencies up to $20.1 \%$ within their short time of development since their emergence. ${ }^{3-7}$ In the case of semitransparent photovoltaic devices, three main areas of focus are recognized in the literature for advancing transparency and performance. First, the strategic selection or design of the absorber material; $;^{8,9}$ second, the realization of light management approaches through device engineering or nanostructured materials and architectures; $\mathbf{1 0}^{\mathbf{1 0}}$ and third, the development of 
semitransparent electrodes, combining efficient charge collection and transparency. ${ }^{11,12}$

The demonstration of high efficiencies $(>20 \%)$, the possibility for low processing temperatures and inexpensive raw materials have positioned perovskite solar cells as promising candidates to replace silicon technologies as the leading architecture for solar energy generation..$^{3-6}$ For these reasons, selecting perovskite-based materials as absorbers for semitransparent photovoltaic devices in order to pursue high efficiencies has become a very attractive strategy. ${ }^{11-16}$ Regarding the correct selection of the electrode, the utilization of sub- $20 \mathrm{~nm}$ layers of $\mathrm{Au}$, often combined with capping layers for protection and reduction of energy loss via device specular reflection, ${ }^{13,16}$ silver nanowires (AgNWs), ${ }^{11,12,24}$ carbon nanotubes (CNTs) ${ }^{15}$ and very recently graphene single layers ${ }^{14}$ has led to promising alternatives.

Structural and morphological optimization of the perovskite layer for semitransparent applications is a less explored topic in the literature. One particular approach is based on the inverted structure $\mathrm{FTO} / \mathrm{TiO}_{2} /$ perovskite/spiro-OMeTAD/Au using a very thin $(10 \mathrm{~nm})$ gold metal film. In the latter work, the concept of 'perovskite islands' was introduced achieving $3.5 \%$ PCE (power conversion efficiency) and an average visible transmittance (AVT) of $\sim 30 \%(370-740 \mathrm{~nm} \text { range) })^{13}$ at best. A limiting trend between the area of the non-uniformly covered absorber film and the corresponding $V_{\mathrm{oc}}$ and fill factor (FF) of the resulting device was evident and attributed to a parallel diode between the electron transporting layer (ETL) and the hole transporting layer (HTL). Attempts to improve the transparency of the perovskite layer through control of the active layer thickness have been reported before, mostly at the cost of severe performance losses. For instance, Peng You et al. reported a drop in $V_{\text {oc }}$ by $\sim 25 \%$, accompanied by a $\sim 10 \%$ loss in FF when decreasing the thickness of the perovskite layer from $350 \mathrm{~nm}$ to $150 \mathrm{~nm}$. A maximum efficiency of 12.05$5.84 \%$ AVT and a minimum of $5.98 \%$ PCE-21.76 AVT were obtained. ${ }^{14}$ Other research groups have also observed similar decreases in $V_{\text {oc }}$ as the perovskite layer film becomes thinner. Specifically, attempts comprising solution based methods, ${ }^{14,17-19}$ thermal evaporation of $\mathrm{PbI}_{2}$ in what was called a two-step deposition method, ${ }^{20}$ and more recently, dipping vapor deposition have all led to significant $V_{\mathrm{oc}}$ losses. ${ }^{21}$ Subsequent studies by means of a thermal co-evaporation process were made with the aim of improving the continuity of the perovskite film and its impact on the resulting efficiency. ${ }^{16} \mathrm{In}$ this way, $V_{\mathrm{oc}}$ values higher or equal to $1 \mathrm{~V}$ with no significant variations were observed using perovskite films as thin as $40 \mathrm{~nm}$ with a striking AVT value of $35 \%$, and a PCE of $3.39 \%$. Whether with the use of high-temperature-based $\mathrm{TiO}_{2}$ as ETL, ${ }^{13,14}$ the use of innovative buffer layers to reduce parasitic absorption in the device stack, ${ }^{22}$ the use of electrodes or active layers based on highly energy-intensive evaporation techniques, ${ }^{13,16,23}$ or the deposition of thinner films leading to inefficient yet highly transparent devices, ${ }^{13,14,16}$ the full potential of perovskite-based semitransparent photovoltaic devices towards large-scale production is a concept that remains not fully attained.
In the present manuscript we establish two processes for the fabrication of semitransparent devices using AgNWs as the electrode material, leading to an efficiency of $7.68 \%$ combined with an AVT of $37 \%$ for the full device (42\% AVT without electrode) for one process, and $3.55 \%$ PCE combined with a record transparency of $46 \%$ AVT ( $50 \%$ AVT, w.o. electrode) for the other process. This result corresponds to an unprecedented combination of optical transparency and maximum power conversion efficiency. Furthermore, by controlling the morphology and thickness of the perovskite layer we are able to tune the shortcircuit photocurrent density $\left(J_{\mathrm{sc}}\right)$ from 5.35 ( $46 \%$ AVT) to a value of $19.10 \mathrm{~mA} \mathrm{~cm}{ }^{-2}$ (14\% AVT), corresponding to a maximum efficiency of $12.95 \%$.

We previously demonstrated the compatibility of AgNWs for processing semitransparent perovskite photovoltaic devices, although these devices still had lower performance and lack in shelf life. ${ }^{12}$ Using the scalable spray coating technique we created an ohmic contact between $\mathrm{ZnO}$ nanoparticles and the AgNW electrode. However, the use of water as the main solvent for the AgNW seriously limited the shelf life time of the resulting solar cells, hindering further optimization of this device architecture. Yet, this work paved the way for further investigation. In this work, by replacing water in the AgNW colloidal solution with isopropyl alcohol we demonstrate AgNW top electrodes that no longer compromised the integrity of the resulting device. We show that the resulting devices feature a shelf life comparable to their counterpart with an evaporated silver electrode. The selection of AgNWs as an alternative to sputtered or thermally evaporated metal electrodes offers the possibility of using solution-processed, high-throughput coating methods which are highly beneficial for the bill of materials (BOM) and the energy pay back time (EPBT) ${ }^{25}$

\section{Results and discussion}

We selected the ITO/PEDOT:PSS/MHP/PC ${ }_{60} \mathrm{BM} / \mathrm{ZnO} /$ electrode structure (where MHP stands for mix halide perovskite, see ESI $\dagger$ for details) due to the potential use of thin ETL and HTL layers, low-temperature processing and hysteresis-free ${ }^{17,26}$ characteristics (the schematic structure of the device configuration and energy diagram can be found in Fig. S1†). With this approach we avoid the widely used and costly spiro-OMeTAD, which typically involves $\sim 300 \mathrm{~nm}$ thick layers, and whose functionality relies upon extra doping steps. In addition, the use of this thick, partially oxidized spiro-OMeTAD layer results in parasitic absorption leading to a reduction of the AVT. ${ }^{16}$ Albeit we recently demonstrated the use of water free PEDOT:PSS as a HTL for environmentally stable inverted perovskite solar cells, again, a very thick layer $(\sim 300 \mathrm{~nm})$ is needed. ${ }^{27}$ Similar to spiroOMeTAD, the latter also contributes to parasitic absorption, limiting the device in terms of transparency.

\subsection{Solvent induced fast crystallization method for opaque devices}

With the aim of producing a compact perovskite layer we applied solvent induced fast crystallization deposition (FCD) $)^{18,28}$ 
combined with nitrogen flow gas-quenching $(\mathrm{GQ})^{29,30}$ during the ordinary precursor based method and a step-like annealing profile (see Fig. S2 and ESI $\dagger$ for details). Using this new approach, whose novelty is based on the correct combination of these three different process conditions, we fabricated opaque devices varying the spin coating speed and the concentration of the precursor solution in order to find an optimum trade-off between photovoltaic parameters and thickness of the active layer (AL) (Table 1). We found PCEs ranging from $10.12 \%$ to $15.99 \%$ corresponding to the $\mathrm{AL}$ of $70 \mathrm{~nm}$ and $340 \mathrm{~nm}$, respectively. Typical $J-V$ curves from best performing devices are depicted in Fig. 1a and b. We observed no significant impact of the film thickness, neither on the FF nor on $V_{\text {occ }}$. The FF values ranged from $78.62 \%$ to $71.70 \%$ and $V_{\text {oc }}$ remains constant among all thicknesses, suggesting that a continuous and robust active layer film could be achieved via FCD-GQ at relatively low annealing temperatures (under $140{ }^{\circ} \mathrm{C}$ ). To further investigate the accuracy of the $J_{\mathrm{sc}}$ obtained from the solar simulator, we recorded the external quantum efficiency $(E Q E)$ of the opaque devices (Fig. 1a). We found an agreement between the $J_{\text {sc }}$ calculated from the $E Q E$ and that obtained from the $J-V$ characterization within $6 \%$ of discrepancy. A key collection of photovoltaic metrics, deduced from Fig. 1, is depicted in Table 1. Additionally, complementary information including statistical relevant parameters can be found in Tables S1 and S2. $\dagger$

The optical density (OD) spectra of bare AL films show an increase in the absorbance as the film thickens, which is in agreement with the integrated $J_{\mathrm{sc}}$ obtained by the $E Q E$ measurements, their corresponding $J-V$ curves (Fig. $1 \mathrm{c}$ and d) and previously reported trends. ${ }^{20,21}$ We note that we observed a thickness limit with our method. For bare AL films deposited at speeds lower than $700 \mathrm{rpm}$, corresponding to thicknesses higher than $340 \mathrm{~nm}$, we observed a downtrend in the OD in the wavelength regime lower than $500 \mathrm{~nm}$ (Fig. S3†). A change in the absorption spectrum suggests a difference in conformation of the resulting perovskite film, most likely due to incomplete crystallization. In light of these findings, we determined that the application of FCD-GQ is revelry limited for devices with perovskite layers exceeding $350 \mathrm{~nm}$, producing low quality, visibly opaque films, suggesting a change in the film roughness (cf. AFM micrographs below). Moreover, the resulting devices denote a significant drop in performance; leading to poor reproducibility and decrease in the shunt resistance and $V_{\mathrm{oc}}$, ranging from 0.5 to $0.7 \mathrm{~V}$ at best (due to lack of statistic consistency on these devices, $J-V$ characterization is not shown).
The EQE spectra of corresponding devices showed a welldefined signature structure with 4 distinct features shifting to higher wavelengths as the thickness of the film increased, most likely attributed to optical interference (Fig. 1d and e). The characteristic optical behavior is in agreement with previously reported finding: $\mathrm{s}^{31}$ and it is attributed to optical interference effects. Moreover, the strong dependence of the $E Q E$ spectral shape on the active layer thickness suggests optically clean interfaces, which is a desirable attribute for a planar PV architecture.

The perovskite films were further analyzed by means of grazing incidence X-ray diffraction (GIXRD) (Fig. 1d). The GIXRD patterns show sharp and strong peaks at $14.18^{\circ}$ and $28.46^{\circ}$, with variations on the full width half maximum (FWHM) below $2 \%$. These peaks are associated with the Miller indices (110) and (220) of the tetragonal phases of perovskites. ${ }^{32-34}$ The GIXRD peak ratio between (110) and (220) was found to be 12.5, $13.9,12.3$ and 11.8 for the films of 339, 139, $\sim 100$ and $70 \mathrm{~nm}$ thick perovskite, respectively. These ratios are in the same range for all the thicknesses, suggesting that no significant compositional or overall crystal orientation change was induced by changing the coating speed or the precursor concentration. Furthermore, the (110): (220) peak ratio observed in films without the implementation of FCD and GQ was considerably decreased to a value of 4 (Fig. S4 $\dagger$ ). As evidenced by the GIXRD study, two main differences can be elucidated compared to the standard method (without FCD-GQ). The first is a strong increase in crystallinity as evidenced by the higher overall peak intensity for films with the same thickness. The second is a strong crystallographic phase orientation, as depicted by the (110) : (220) intensity ratios. These two characteristics redound to better performance and the possibility of tuning the resulting $J_{\mathrm{sc}}$ by changing the thickness of the AL without jeopardizing $V_{\mathrm{oc}}$ or FF. The peaks at $35^{\circ}$ and $51^{\circ}$ are characteristic of the underlying ITO layer, also explaining their intensity gain as the film becomes thinner (Fig. S4†). The pattern associated with the presence of PEDOT:PSS is screened in the case of the thicker layer, being observable at $30^{\circ}$ only in the films with 139,100 and $70 \mathrm{~nm}$ thick perovskite (Fig. S4†).

In order to study differences in the morphology we performed atomic force microscopy (AFM) on films with increasing thickness. As is apparent from the micrographs in Fig. 2, the well-defined micro-structured domains increase markedly in size with increasing film thickness. Our method leads to grain sizes as large as 6.5 microns for the thicker films and as small as

Table 1 Key metrics for best performing opaque (OP) devices using the FCD-GQ method ${ }^{b}$

\begin{tabular}{llllll}
\hline ID & Thickness $(\mathrm{nm})$ & $E Q E J_{\mathrm{sc}}\left(\mathrm{mA} \mathrm{cm}^{-2}\right)$ & $J_{\mathrm{sc}}\left(\mathrm{mA} \mathrm{cm}^{-2}\right)$ & $V_{\mathrm{oc}}(\mathrm{V})$ & $\mathrm{FF}(\%)$ \\
\hline FCD-OP 1 & $70 \pm 14$ & 14.59 & 15.91 & 0.96 & 75.81 \\
FCD-OP 2 & $100 \pm 17$ & 15.38 & 16.27 & 0.96 & 73.63 \\
FCD-OP 3 & $129 \pm 18$ & 17.54 & 18.79 & 0.94 & 74.55 \\
$\vdots$ & $\vdots$ & $\vdots$ & $\vdots$ & $\vdots$ & $\vdots$ \\
FCD-OP 7 & $339 \pm 16$ & 21.93 & 22.05 & 0.99 & $\vdots$ \\
\end{tabular}

${ }^{a}$ PCE values are calculated using $J_{\mathrm{sc}}$ extracted from EQE measurements. ${ }^{b}$ Complete collection of the metrics along with complementary information including statistical relevant parameters can be found in Tables S1 and S2. 
a)

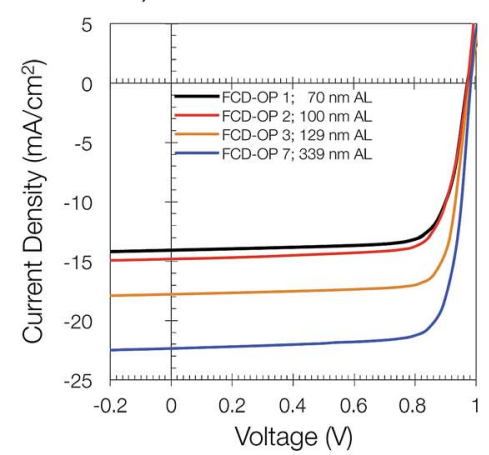

d)

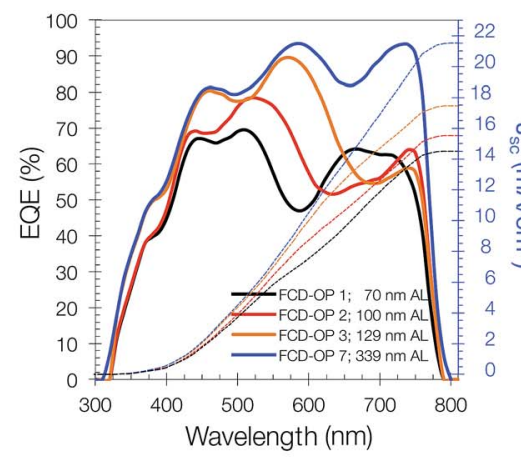

b)

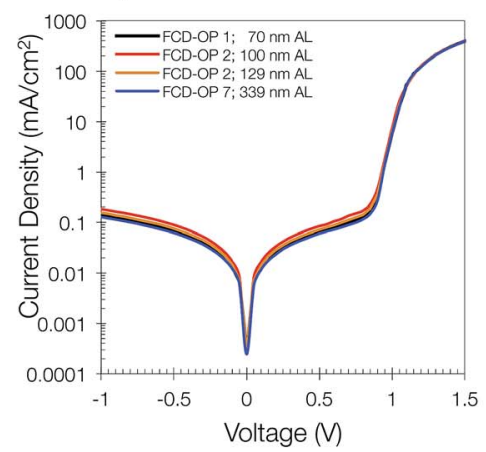

e)

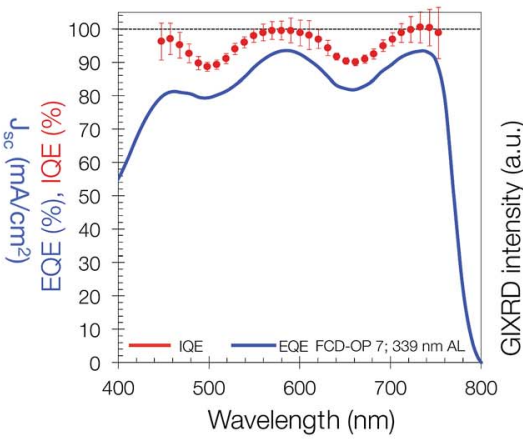

c)

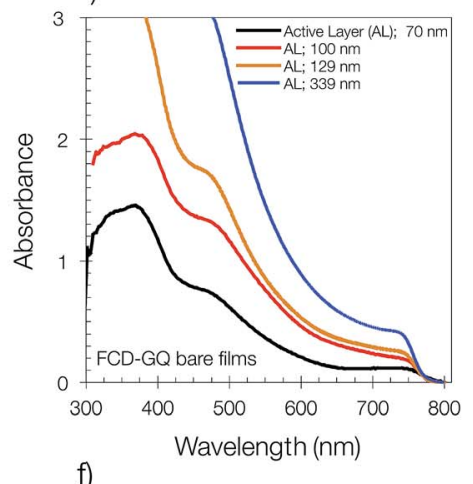

f)

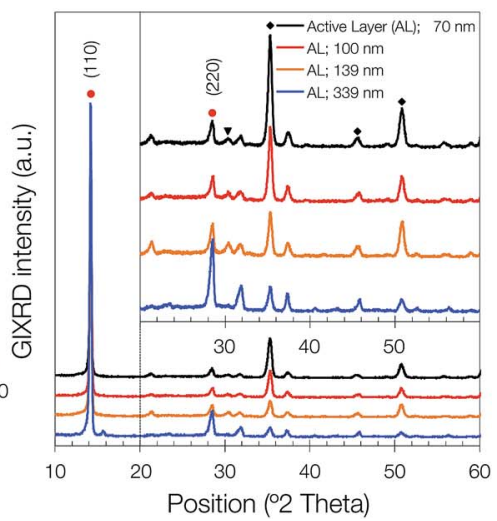

Fig. 1 (a) $J-V$ characteristics under $A M 1.5$ irradiation at $0.1 \mathrm{~W} \mathrm{~cm}^{-2}$ illumination of devices with an average FF as a function of active layer thickness; (b) corresponding dark current; (c) absorption spectra of bare active layer films produced with the FCD-GQ method; (d) EQE spectra of opaque devices with increasing active layer thickness; (e) IQE evaluation of the best performing perovskite opaque device by the FCD-GQ method. (f) GIXRD spectra of bare films produced by the FCD-GQ method.

0.86 microns for the thinner films. Consequently, the roughness (rms) of the resulting films increased from $14.0 \mathrm{~nm}$ to 48.3 $\mathrm{nm}$ for the 70 and $339 \mathrm{~nm}$ films, respectively. However, this increase in roughness did not have an impact on device performance, showing a maximum $\mathrm{FF}$ of $76.06 \%$ for devices fabricated with $339 \mathrm{~nm}$ thick-48.3 rms films. We further evaluated the morphology of the large- and small-perovskite domains in full devices through cross-sectional imaging by means of high resolution scanning electron microscopy (SEM). A strong contrast between the different layers and the well-defined edges indicates no obvious interlayer diffusion. In the case of thinner films, several cavities throughout the AL that appear to be hollow were observed (Fig. 3, label no. 1). We therefore propose that in the case of ultra-thin films, these hollow spaces are generated as a result of the fast acceleration speed in the early stages of deposition. Owing to this rapid acceleration, the contact time of the exhaust volume with the underlying PEDOT:PSS layer is not sufficient to spread evenly, hence leaving uncovered areas that will later turn into caves. Different features on the cross-sectional micrographs of thicker films (238 nm and $339 \mathrm{~nm}$ ) can be recognized. The surface topography of the perovskite layers shows a step-like sharp profile. This step-like topography can be associated with the domain edges shown by the AFM micrographs, being comparable in size and shape (Fig. 3, solid red line). Given the fact that the contrast in electron microscopy correlates with the electron density, it can be depicted by label number 2 in Fig. 3 (bright contrast) what appears to be the formation of small domains with higher electron density. These small features at the interface suggest a change in the compositional atomic mass, surface topography and potentially crystal orientation, owing to the implementation of FCD and GQ. During the FCD, with the addition of a copious amount of chlorobenzene in the final stages of the deposition, the solubility of the precursor at the surface is reduced significantly, thereby, promoting fast nucleation and growth of more oriented crystals. Subsequently, the function of the nitrogen GQ is to further stimulate nucleation along with rapid crystal growth during a drying/supersaturation stage. The latter facilitates the formation of the observed large domains. In this way, we imagine that the growth of crystalline domains is only limited by the volume of the precursor available and its concentration, hence determined by the speed of the deposition, which also defines the final thickness of the film. This explains why the domain size observed in the AFM micrographs becomes larger as the speed of the deposition is decreased. A third feature in the form of round "bubble-like" spots can be distinguished in the SEM cross-sections. We attributed the presence of these spots to a measurement artifact, since we observed them appear and disappear during the measurement. It is important to emphasize that the features depicted by label 

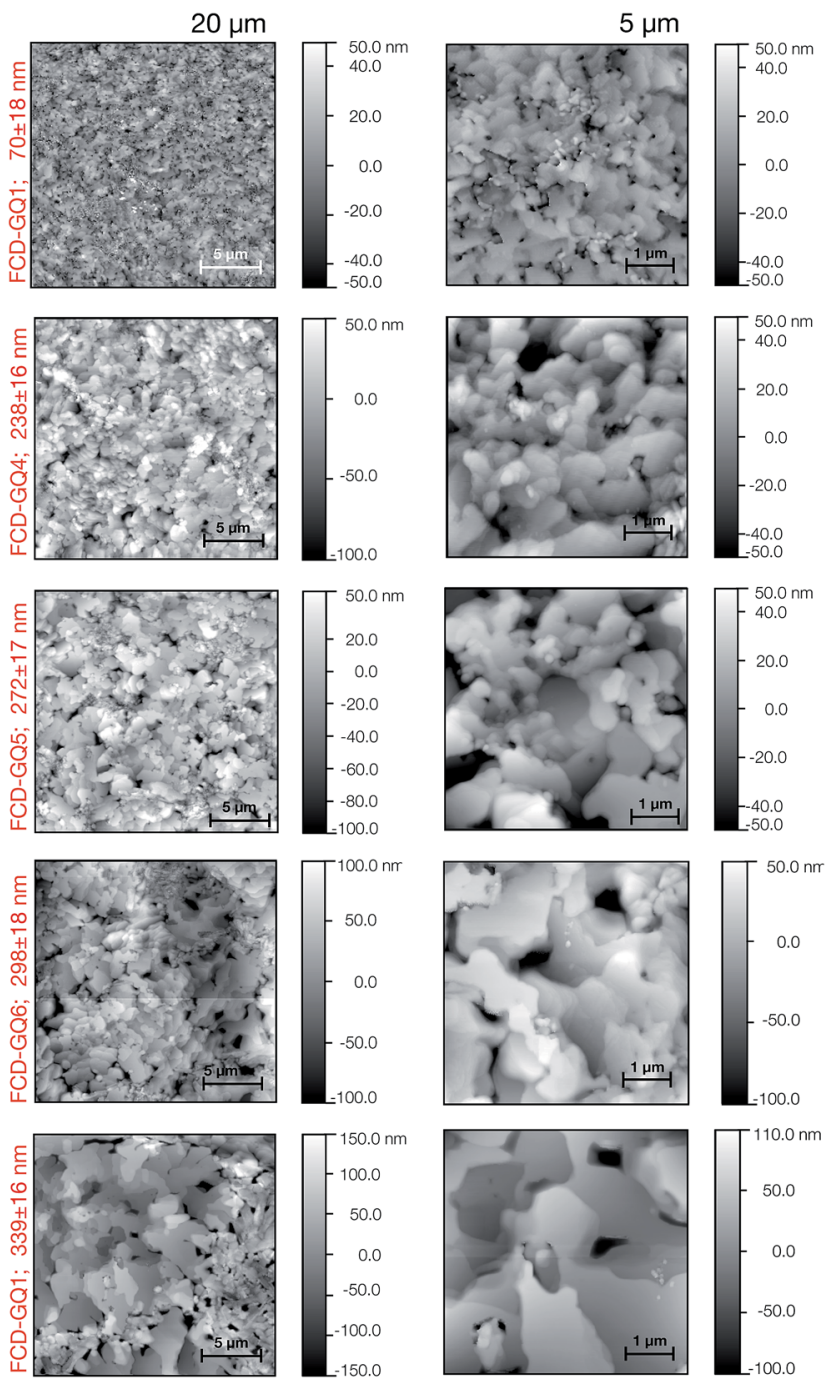

Fig. 2 Topography of $\mathrm{MAPbl}_{3-x} \mathrm{Cl}_{x}$ films with different active layer thicknesses as measured by intermittent contact atomic force microscopy (AFM). The column on the left shows $20 \mu \mathrm{m} \times 20 \mu \mathrm{m}$ areas. The column on the right shows $5 \mu \mathrm{m} \times 5 \mu \mathrm{m}$ area.

2 at the top interface of the perovskite layers did not change/ emerge during the measurement.

\subsection{Solvent induced fast crystallization method for semitransparent devices}

After demonstrating a new and simple protocol for the deposition of ultra-thin perovskite films without hampering the resulting performance, our further interest was to elaborate semitransparent devices by employing AgNW electrodes instead of evaporated silver. By using the spray coating technique in air, we deposited a thin layer of AgNWs on the top of the ZnO buffer layer (Fig. S1 $\dagger$ ). The utilization of a mask allowed defining the active area. The final deposited AgNW film had a total transmittance of $\sim 85 \%$ at $550 \mathrm{~nm}$. In order to control the sheet resistance of the coated AgNW electrode, we coated glass substrates along with the solar cells and measured the sheet resistance using the four-point probe technique. The measured
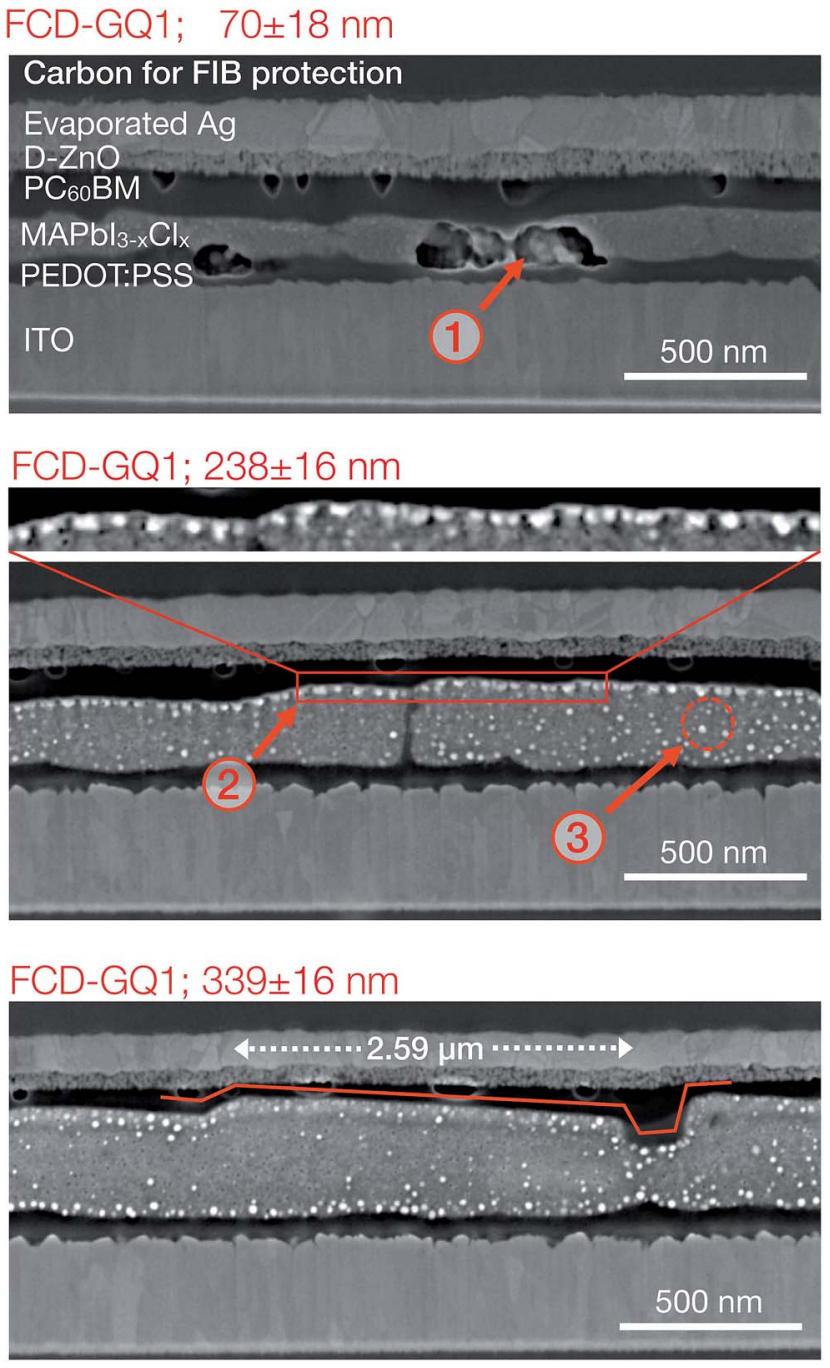

Fig. 3 Cross-sectional scanning electron microscopy (SEM) micrographs of full opaque devices. Labels 1, 2 and 3 point out different features or artifacts in the film (see text for details). The specifications of the microscope for all SEM image acquisitions were: thermoluminescent dosimeter (TLD) detector with a magnification of $65000 \times$, a current of $0.10 \mathrm{nA}$, a dwell of $500 \mathrm{~ns}, \mathrm{HFV} 3.9 \mu \mathrm{m}$ and a HW of 3.7 $\mathrm{mm}$.

sheet resistance of the reference AgNW-coated glass ranged between $10 \mathrm{ohms} \mathrm{sq}^{-1}$ and $20 \mathrm{ohms} \mathrm{sq}^{-1}$. We found that these variations in sheet resistance have a negligible impact on the device performance. We further observed, through control experiments, that the short exposure times of the half-cell (solar cell stack without the electrode) to the environment $t$ (20-30 minutes, $40 \% \mathrm{RH}$ at room temperature) did not have a negative impact on the device. The ZnO buffer layer was activated with a short exposure time $(10 \mathrm{~s})$ to UV radiation. ${ }^{35}$

Current-voltage curves of best performing semitransparent devices can be seen in Fig. 4a and b. A summary of key parameters is presented in Table 2. In addition, a complete collection of transparency parameters along with complementary statistics for device evaluation is shown in Tables S3 and S4. $\dagger$ Similar to its opaque counterparts, the $J_{\mathrm{sc}}$ drawn from the 
a)

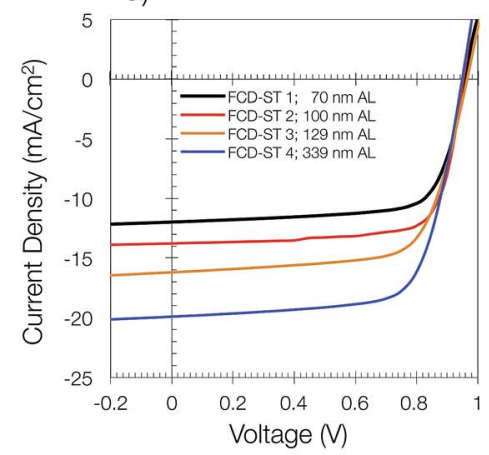

d)

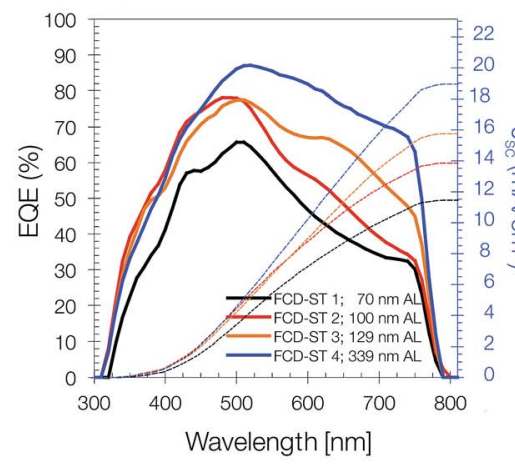

b)

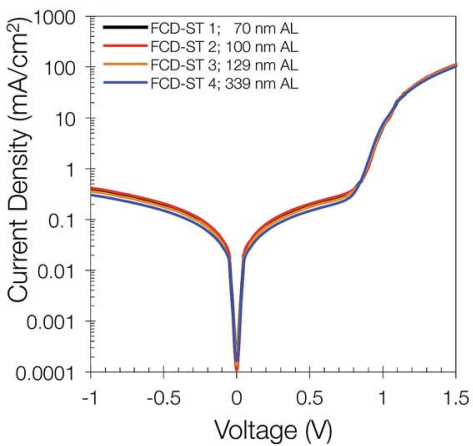

e)

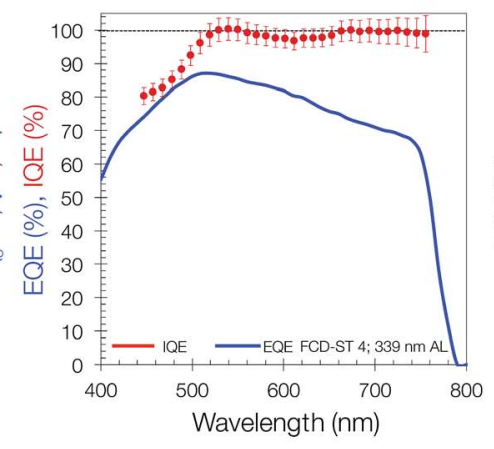

c)

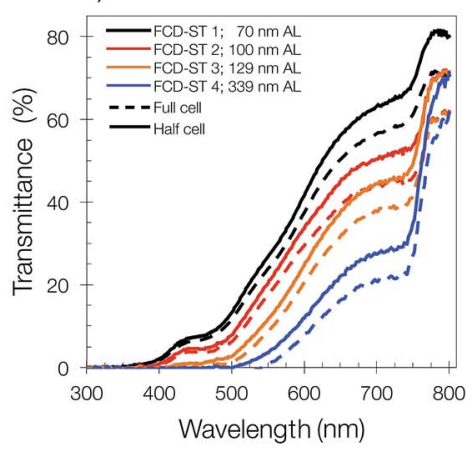

f)

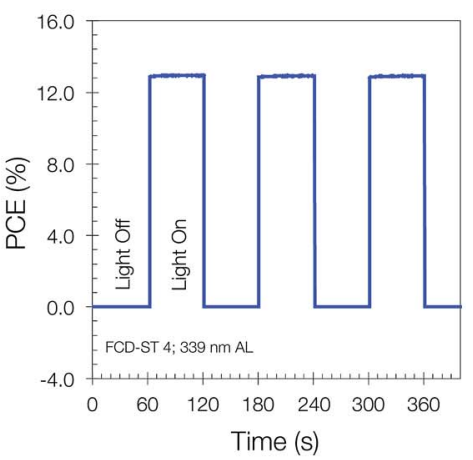

Fig. 4 (a) J-V characteristics under AM 1.5 spectrum at $0.1 \mathrm{~W} \mathrm{~cm}{ }^{-2}$ illumination of devices with an optimum FF as a function of active layer thickness; (b) corresponding dark current; (c) total transmittance spectra of full devices with the top AgNW electrode and without the top AgNW electrode; (d) EQE spectra of semitransparent devices with increasing thickness; (e) IQE evaluation of the best performing perovskite opaque device by the FCD-GQ method; (f) photocurrent response of semi-transparent device FCD-ST 4 for three light on-off switching cycles.

electrical characterization is consistent with the optical properties of the AL and with the $E Q E$ measurements (Fig. 4a and 1e). As can be seen from the $E Q E$ spectral shape (Fig. 4a and c), the absence of the back electrode is evident for most of the cases and results in a lower $J_{\mathrm{sc}}$, showing the characteristic nonreflective nature of the AgNW electrode. As expected, this effect is more significant for the thinner $(70 \mathrm{~nm})$ perovskite films, where a larger fraction of photons impinging on the AL remains unabsorbed, leading to a more pronounced drop in $J_{\mathrm{sc}}$. The resulting efficiencies ranged from $7.81 \%$ to $\sim 37 \%$ AVT to $12.78 \%$ to $\sim 18 \%$ AVT. The total transmittance profile of all devices with and without the electrode is shown in Fig. 4c. The AVT without the electrode reached $42 \%$ for the solar cells with thinner $(70 \mathrm{~nm})$ AL films ( $27 \%$ of transmittance at $550 \mathrm{~nm})$. The
$J_{\text {sc }}$ losses as compared with the same device geometry using evaporated silver were $27.54 \%, 9.93 \%, 2.57 \%$ and $14.67 \%$ for the films of $70 \mathrm{~nm}, 100 \mathrm{~nm}, 129 \mathrm{~nm}$ and $340 \mathrm{~nm}$, respectively, i.e., we observe an optimum AL thickness of $129 \mathrm{~nm}$ for optimum current generation/extraction in combination with an $\mathrm{AgNW}$ electrode. Moreover, the observed FF was overall constant within the statistical error (Table S4 $\uparrow$ ). We obtained a general drop in $\mathrm{FF}$ of $6 \%$ when employing AgNWs as compared with evaporated silver. The small impact on the FF can be attributed to the utilization of the ultra-thin ETL, HTL and buffer layers, allowing efficient charge extraction and transfer at low optical losses. Moreover, we infer that the highly oriented perovskite crystals allow optimum charge extraction at the $\mathrm{AL} / \mathrm{ETL}$ interface. In order to analyze any transient

Table 2 Key metrics for best performing semitransparent devices using the FCD-GQ method ${ }^{d}$

\begin{tabular}{|c|c|c|c|c|c|c|c|}
\hline ID & Thickness (nm) & $E Q E J_{\mathrm{sc}}\left(\mathrm{mA} \mathrm{cm}^{-2}\right)$ & $V_{\mathrm{oc}}(\mathrm{V})$ & $\mathrm{FF}(\%)$ & $P C E^{a}$ & $\operatorname{AVT}^{b}(\%)$ & $\operatorname{AVT}^{c}(\%)$ \\
\hline FCD-ST 1 & $70 \pm 14$ & 11.55 & 0.97 & 72.23 & 7.81 & 42 & 37 \\
\hline FCD-ST 2 & $100 \pm 17$ & 14.23 & 0.98 & 72.07 & 9.55 & 33 & 29 \\
\hline FCD-ST 4 & $339 \pm 16$ & 19.10 & 0.97 & 70.85 & 12.95 & 18 & 14 \\
\hline
\end{tabular}

${ }^{a} P C E$ values are calculated using $J_{\mathrm{sc}}$ extracted from $E Q E$ measurements. ${ }^{b}$ Average visible transmittance of devices without top electrode. ${ }^{c}$ Average visible transmittance of devices with top electrode. ${ }^{d}$ Complete collection of metrics along with complementary information including statistical relevant parameters can be found in Tables S3 and S4. 
photovoltaic effect in the semitransparent devices we measured the power conversion efficiency $(P C E)$ over time. In Fig. $4 \mathrm{f}$ we show a representative $P C E$ trace $v s$. time during cyclic illumination. The voltage at the maximum power point $\left(V_{\max }\right)$ and the measured photocurrent density at the maximum power point $\left(J_{\max }\right)$ define the $P C E$ over time. The $V_{\max }$ interpolation is done on $J-V$ curves obtained every second through a $V_{\max }$ tracking algorithm. The quick transient photocurrent response further suggests no hysteretic effect in our semitransparent devices. In addition, we did not observe any decay in the steady-state response of the devices, suggesting a robust charge extraction mechanism (Fig. S5†). These results inform that water free AgNW electrodes feature suitable optical and electrical characteristics as well as high chemical compatibility with the perovskite layer to form efficient and robust semitransparent photovoltaic devices.

In addition, in Fig. 1e and 4e we present the internal quantum efficiency (IQE) evaluation of the best performing opaque and semitransparent devices, respectively. The IQE was derived using a systematic approach reported elsewhere. ${ }^{36,37}$ In order to consider the electro-optical cavity effects in the IQE calculation we performed accurate total reflectance and transmittance measurements along with a transfer-matrix assessment of all non-active parasitic absorbances including the AgNW electrode for the semitransparent devices (schematic workflow depicted in Fig. $\mathbf{S} 7 \dagger$ ). The resulting spectral shape of the $I Q E$ of the opaque device is in agreement with previously reported results within the limitations of the experimental error. ${ }^{31,38}$ The IQE spectral shape shows an overall semi-flat behavior and in the vicinity of $100 \%$ for both architectures showing no significant influence of hot exciton or charge carrier effects as well as no energy dependent charge generation. Likewise, this analysis further confirms the almost perfect charge generation and subsequent collection of our perovskite devices. Additionally, for the case of the semitransparent devices it also confirms the efficiency and robustness of the AgNW as an alternative electrode.

\subsection{Solvent-solvent extraction method for semitransparent devices}

To overcome the transparency limitations given by the utilization of the FCD-GQ method, we developed a new protocol based on the solvent-solvent extraction method (SSE). During the SSE method, the partially dry perovskite film is rapidly submerged into an extraction solvent. The high miscibility between the precursor-solvent and the extraction-solvent allows diffusion of the solvent contained in the film, thus inducing crystallization. During crystallization, the definition of the chemical environment with the correct selection of solvents is crucially important for crystal growth. ${ }^{39}$ Commonly used precursor solvents for the solution process preparation of perovskite-based solar cells are $\gamma$-butyrolactone (GBL, dielectric constant, $\varepsilon=39.1$ ), $N, N$-dimethylformamide (DMF) $(\varepsilon=38.3)$ and dimethylsulfoxide (DMSO) $(\varepsilon=47.2)$, due to their high solubility and moderate coordination properties. Crystallization of $\mathrm{CH}_{3} \mathrm{NH}_{3} \mathrm{PbI}_{3}$ with the use of $\gamma$ butyrolactone as the precursor solvent has successfully led to high quality single crystal growth by means of the vapor-assisted crystallization method. ${ }^{\mathbf{4 0}}$ Furthermore GBL does not induce adduct formation with $\mathrm{PbI}_{2}$ like in the case of DMSO $\left(\mathrm{PbI}(\mathrm{DMSO})_{2}\right)$, which will retard the crystallization when performed at room temperature, potentially leading to poor film quality. ${ }^{41,42}$

In the present case, we explored $\gamma$-butyrolactone $\left(\varepsilon_{\mathrm{GBL}}=39.1\right.$, $\mathrm{bp}_{\mathrm{GBL}}=204^{\circ} \mathrm{C}$ ) as the precursor solvent due to its high boiling point and dielectric constant (see the Experimental section for further details). During this process, the perovskite film is rapidly submerged either in toluene, $\mathrm{TE}\left(\varepsilon_{\mathrm{TE}}=2.38, \mu_{\mathrm{TE}}=0.36\right.$ D) or in diethyl ether, DEE $\left(\varepsilon_{\mathrm{DEE}}=4.20, \mu_{\mathrm{DEE}}=1.15 \mathrm{D}\right)$ as the extraction solvent to induce fast crystallization. Both toluene and diethyl-ether have been previously reported mainly for controlled precipitation of $\mathrm{CH}_{3} \mathrm{NH}_{3} \mathrm{PbI}_{3}$ perovskite micro- and nano-particles extracted from GBL precursor solution. ${ }^{43}$

As apparent from Fig. 5a and S8 and ESI video, $\uparrow$ the deposited precursor changes color almost instantly when in contact with the extraction solvent. The precursor film darkens within the first 5 seconds. Notably, the use of toluene induces faster change in color compared to diethyl ether, potentially due to incomplete crystallization in the case of diethyl ether.

The rapid nature of the crystallization process (supersaturation, nucleation and crystal growth under 5 seconds) facilitates full deposition of all layers under ambient conditions in the presence of a relatively high humidity (45\% $\mathrm{RH})$ and with good reproducibility (Table S5 and Fig. S6†). Zhou et al. ${ }^{\mathbf{4 4}}$ reported a similar approach with the utilization of $N$-methyl-2pyrrolidone (NMP, $\varepsilon_{\mathrm{NMP}}=39.1$, bp $\left.\mathrm{NMP}_{\mathrm{NM}}=202{ }^{\circ} \mathrm{C}\right)$ as the precursor solvent and DEE as the extraction solvent. However, in that case the crystallization of $\mathrm{CH}_{3} \mathrm{NH}_{3} \mathrm{PbI}_{3}$ took about $2 \mathrm{~min}$. In our case, we observed a much shorter crystallization time (under 5 seconds) while still producing high quality and homogeneous thin perovskite films.

Using toluene and DEE as extraction solvents in the solventsolvent extraction method, we were able to fabricate devices

Table 3 Key metrics for best performing semitransparent devices using the SSE method using toluene as the extraction solvent ${ }^{d}$

\begin{tabular}{|c|c|c|c|c|c|c|c|}
\hline ID & Thickness (nm) & $E Q E$, sol. sim. ${ }^{a} J_{\mathrm{sc}}\left(\mathrm{mA} \mathrm{cm}{ }^{-2}\right)$ & $V_{\mathrm{oc}}(\mathrm{V})$ & $\mathrm{FF}(\%)$ & $P C E^{a}$ & $\operatorname{AVT}^{b}(\%)$ & $\operatorname{AVT}^{c}(\%)$ \\
\hline SSE-TE 1 & $100 \pm 14$ & $9.55,11.22$ & 1.03 & 71.77 & 8.12 & 33 & 28 \\
\hline SSE-TE 2 & $50 \pm 17$ & $4.37,5.35$ & 1.03 & 65.55 & 3.55 & 50 & 46 \\
\hline
\end{tabular}

${ }^{a} P C E$ values are calculated using short circuit photocurrent extracted from $J-V$ characterization AM 1.5 irradiation at $0.1 \mathrm{~W} \mathrm{~cm}^{-2}$ illumination.

${ }^{b}$ Average visible transmittance of devices without the top electrode. ${ }^{c}$ Average visible transmittance of devices with the top electrode.

${ }^{d}$ Complete collection of metrics along with complementary information including statistical relevant parameters can be found in Table S5. 
a)

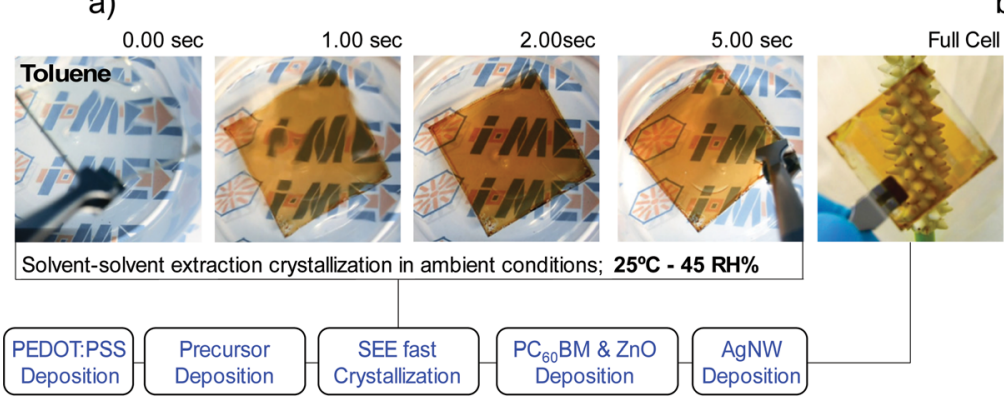

c)

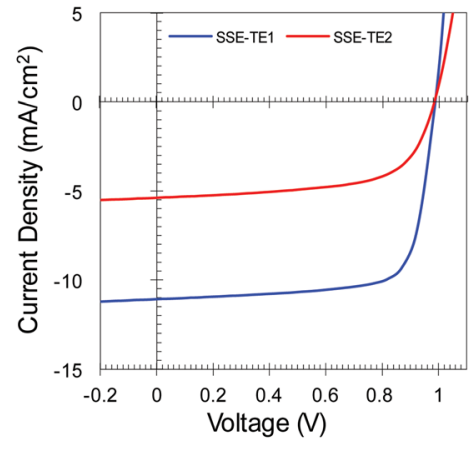

d)

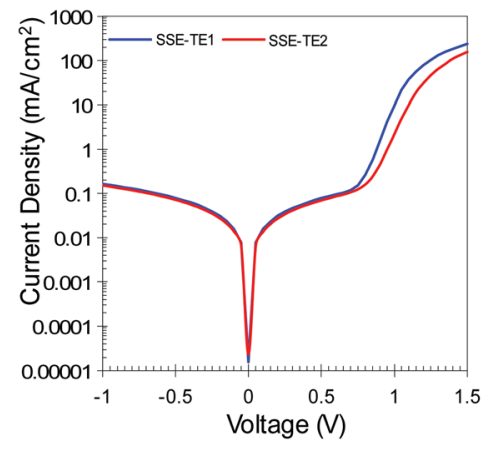

b)

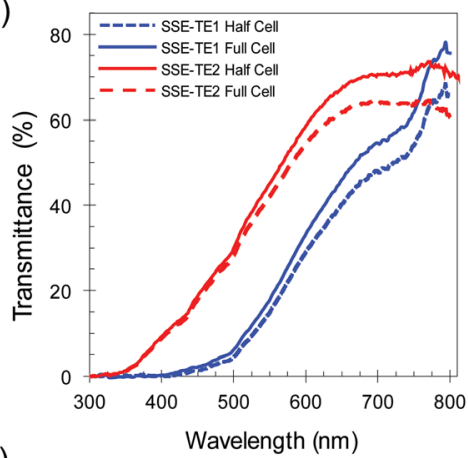

e)

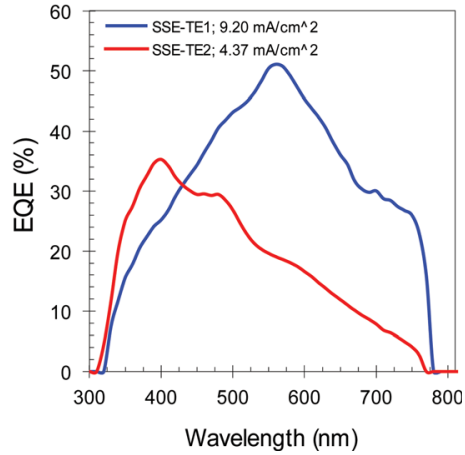

Fig. 5 (a) Schematic representation of the SEE method showing 5 seconds crystallization and final appearance of a full semitransparent cell, no extra annealing of the perovskite film is needed; (b) total transmittance spectra of full devices with the top AgNW electrode and without the top AgNW electrode; (c) J-V characteristics under AM 1.5 spectrum at $0.1 \mathrm{~W} \mathrm{~cm} \mathrm{~cm}^{-2}$ illumination of devices with an optimum FF; (e) $E Q E$ spectra of semitransparent devices using the SEE-TE method.
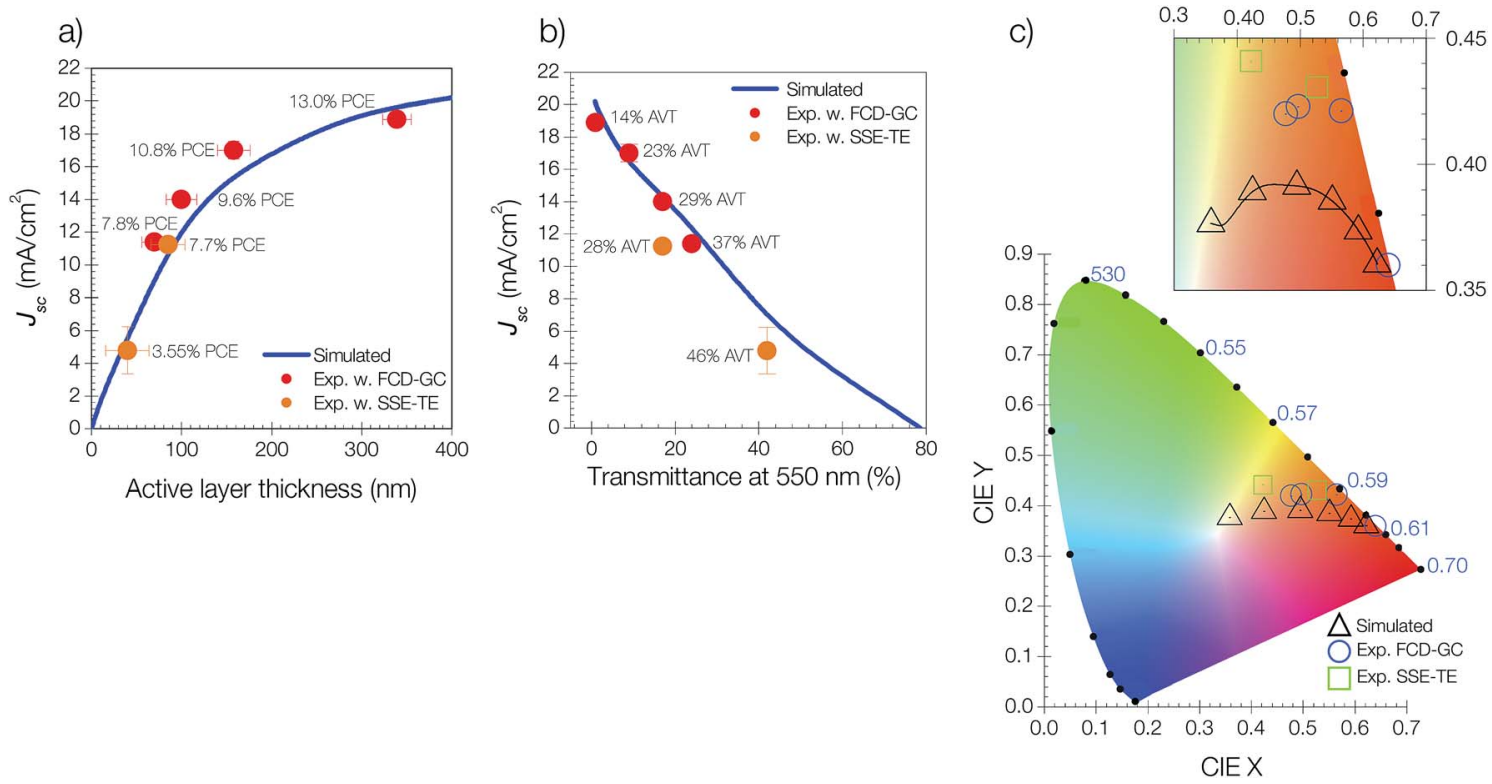

Fig. 6 (a) Simulated behavior of the short circuit photocurrent $\left(J_{s c}\right)$ as a function of active layer thickness; (b) simulated transmittance at 550 nm as a function of $J_{\mathrm{sc}}$. The $J_{\mathrm{sc}}$ utilized for these plots was extracted from $J-V$ characterization AM 1.5 irradiation at $0.1 \mathrm{~W} \mathrm{~cm} \mathrm{~cm}^{-2}$ illumination. (c) Color coordinates of the full semitransparent perovskite solar cell plotted on the CIE xy 1931 chromatic diagram showing: devices fabricated by the FCD-GQ method under inert atmosphere (blue circle), devices fabricated by the SSE-TE method under ambient conditions $\left(25{ }^{\circ} \mathrm{C}, 45 \% \mathrm{RH}\right.$ ) (green square), and simulated values with thicknesses ranging from $0 \mathrm{~nm}$ to $500 \mathrm{~nm}$ in intervals of $100 \mathrm{~nm}$, solid black line and black triangle, respectively. 
with comparable performance than with fast crystallization deposition (Fig. 6). However, using the solvent-solvent extraction method, particularly in the presence of toluene (SSE-TE), we observed significantly better photovoltaic performances when fabricating ultrathin perovskite films $(40 \mathrm{~nm})$ as compared to fast crystallization deposition (Fig. S9†). By applying SSE-TE we were able to fabricate devices with an unprecedented $46 \%$ AVT for a full device stack, corresponding to $45 \%$ total transmittance at $550 \mathrm{~nm}$, while yielding a PCE of $3.6 \%$ based on a $\mathrm{FF}$ of $65.6 \%$, and a $V_{\mathrm{oc}}$ of $1.03 \mathrm{~V}$. Currentvoltage curves of best performing semitransparent devices along with their $E Q E$ profiles and total transmittance spectra are depicted in Fig. 5. A summary of the key parameters is presented in Table 3. In addition, a complete collection of transparency values along with complementary statistics for device evaluation is shown in Tables S5 and S6.†

Finally, in order to correlate transparency and color perception as well as provide a global perspective of our results, we simulated trends for the short-circuit photocurrent as a function of total transmittance at $550 \mathrm{~nm}$ (Fig. 6a) and as a function of active layer thickness (Fig. 6b). Additionally, we calculated and plotted the color coordinates of empirical and simulated semitransparent perovskite solar cells in a CIE $x y$ 1931 chromatic diagram (Fig. 6c). The simulated and experimental short-circuit current data agree closely, confirming the validity of the simple layer optical constants (Fig. S10†). With the combination of the presented experimental methods and optical simulations we are able to cover a wide range of thicknesses and transparencies for perovskite devices, therefore, opening a plurality of potential applications. Since, in addition to modular transparency, aesthetic or complete neutral-color appearance is a desired property for building integration applications, we simulated the color coordinates for a broad range of devices with the active layer thickness extending from $0 \mathrm{~nm}$ to $500 \mathrm{~nm}$ (Fig. 6c). Most importantly and as desired for building integrated photovoltaics (BIPV), the chromaticity coordinates corresponding to the sample SSS-TE2 are located in the vicinity of the neutral zone of the CIE diagram, which positions perovskites as a very competitive technology for the BIPV market.

\section{Conclusions}

In conclusion, we developed a systematic approach for the preparation of highly transparent and highly efficient solar cells using water free AgNW electrodes. We fabricated semitransparent perovskite solar cells at relatively low temperatures utilizing a process that is fully solution based and without being afflicted by hysteresis. We introduced a methodology based on a combination of solvent induced fast crystallization and gas quenching in order to assist the perovskite crystallization process during the later stages of deposition. We infer that this methodology leads to highly oriented crystals, presumably extending to the interface, where efficient charge extraction can occur. Furthermore, we introduce a solvent-solvent extraction protocol to increase the dynamic transparency range of the studied architecture. This protocol allows complete device fabrication under ambient conditions even under relatively high humidity conditions including complete crystallization of the perovskite precursor deposited layer within a 5 seconds period of time and it does not require any type of annealing. Moreover, by associating photovoltaic parameters, such as open circuit voltage and fill factor, with the quality of the perovskite layer, we demonstrated the elaboration of a highly compact, pinhole-free and ultra-thin active layer, allowing us to fabricate semitransparent solar cells with an unprecedented average visible transparency of $46 \%$ combined with an efficiency of $3.55 \%$, based on a high fill factor of $65.55 \%$ and an open circuit voltage of 1.03 (see Fig. S11†). We demonstrated the technical relevance of our method using a robust statistical analysis, showing excellent reproducibility. Hence, this study successfully realizes a robust perovskite-based absorber with elevated extinction coefficient in an ultra-thin configuration without jeopardizing photovoltaic performance and, therefore, leads the way for a successful implementation in high throughput printing technologies.

\section{Materials and methods}

\subsection{Materials and preparation}

Unless stated otherwise, all materials were used as received and were purchased from Merck or Aldrich. Lead(III) chloride ultradried 99.99\% ampouled under argon was purchased from Alfa Aesar and $\mathrm{CH}_{3} \mathrm{NH}_{3} \mathrm{I}$ was provided by Dyenamo. NeutralPEDOT:PSS (Clevios SCA 228) was provided by Heraeus. Nanograde provided $\mathrm{ZnO}$ nanoparticle ink; anhydrous solvents and short exposures to air were used during its preparation upon request. The DMF-perovskite precursor solution was made by adding $\mathrm{PbCl}_{2}$ and $\mathrm{CH}_{3} \mathrm{NH}_{3} \mathrm{I}$ powders with a molar ratio of $1: 3$ and a concentration of $40 \mathrm{wt} \%$ and $30 \mathrm{wt} \%$ to a vial and mixed with anhydrous dimethylformamide (DMF). The solution was then stirred for 30 minutes at $60{ }^{\circ} \mathrm{C}$ and filtered prior to deposition. The GBL-perovskite precursor was made by adding $\mathrm{PbI}_{2}$ and $\mathrm{CH}_{3} \mathrm{NH}_{3} \mathrm{I}$ powders with a molar ratio of approximately $1: 1$ and a concentration of $40 \mathrm{wt} \%$ and $20 \mathrm{wt} \%$ to a vial and mixed with anhydrous gamma-butyrolactone (GBL). The solution was then stirred for 30 minutes at $60{ }^{\circ} \mathrm{C}$ and filtered prior to deposition. Isopropanol based AgNW ink with a $1 \mathrm{wt} \%$ load was provided by Rent A Scientist (RAS). Individual solvents comprising the ink were tested to evaluate whether the perovskite film can withstand its exposure, this would be evident by a change in color from dark brown to light yellow. Acetone, ethylene glycol, isopropanol and ethyl acetate were tested from which only ethylene glycol proved to be detrimental to the film by this particular test. This solvent was removed from the ink upon request. The AgNW ink was used as received.

\subsection{Device fabrication}

Laser patterned ITO (with a roughness of 5-7 rms) substrates were ultra-sonically cleaned using toluene, acetone and isopropanol for 10 minutes each followed by an oxygen plasma cleaning process. The cleaned substrate was then coated with 
a PEDOT:PSS layer by means of spin coating at a speed of 2000 rpm and annealed at $140{ }^{\circ} \mathrm{C}$ for 15 minutes.

After perovskite deposition, a compact $<60 \mathrm{~nm}$ thick layer of $\mathrm{PC}_{60} \mathrm{BM}$ is spin coated. A $2 \mathrm{wt} \%$ solution of $\mathrm{PC}_{60} \mathrm{BM}$ in $\mathrm{CB}$ is then deposited using a three step speed profile with no subsequent annealing. The $\mathrm{ZnO}$ film was spin coated at $2000 \mathrm{rpm}$ and annealed during 5 minutes at $80{ }^{\circ} \mathrm{C}$. For the opaque devices, the counter electrode was deposited through a shadow mask by thermal evaporation under a vacuum of $10^{-6}$ torr. For the semitransparent devices the AgNW counter electrode was deposited using an automated spray coating system. The obtained thick AgNW film had a sheet resistance of around 10-20 ohm sq ${ }^{-1}$ and a transmittance of $<85 \%$ at $550 \mathrm{~nm}$.

FCD-GQ method. Once at room temperature, the substrates were coated with the DMF-perovskite precursor solution with different speeds in order to control the thickness. Two to five seconds before the end of the deposition the samples were drop-cast with $300 \mu \mathrm{L}$ of chlorobenzene and right after the washing step the sample is readily dried with a nitrogen flow during 10 seconds. Subsequently, the samples were kept on a hot plate at a temperature of $50{ }^{\circ} \mathrm{C}$ during a period of approximately 20 minutes followed by a profiled temperatureannealing step (Fig. S2†), all depositions were performed under inert nitrogen atmosphere.

Solvent-solvent extraction method. After PEDOT:PSS deposition, the samples were deposited with the GBL-perovskite precursor via spin coating. The concentrations were $40 \mathrm{wt} \%$ and $80 \mathrm{wt} \%$ for SSE-TE1 and SSE-TE2, respectively. Similarly, the spin coating times and final speeds were, 10 seconds $-4000 \mathrm{rpm}$ and 5 seconds - $6000 \mathrm{rpm}$ for SSE-TE1 and SSE-TE2, respectively. Right after deposition, the samples were submerged into $40 \mathrm{~mL}$ of anhydrous toluene for an average time of 5 seconds; all depositions were performed under ambient conditions $\left(25^{\circ} \mathrm{C}\right.$ and $45 \% \mathrm{RH})$.

\subsection{Device characterization}

Current density-voltage $(J-V)$ characterization under light was carried out by means of a measurement unit from BoTest using a Newport Sol1A solar simulator with an AM 1.5 G spectrum at $0.1 \mathrm{~W} \mathrm{~cm}^{-2}$, which was determined by using a calibrated singlecrystal standard Si-cell. To avoid current contribution from adjacent pixels during the measurement we utilized a shadowing mask for all $J-V$ characterization. External quantum efficiency $(E Q E)$ spectra were recorded with an Enli Technology QE$\mathrm{R}$ measurement system, also calibrated with a Si PV cell. Crosssections of the samples were obtained with a FEI Helios NanoLab 660 DualBeam FIB system. In order to prepare the samples, a carbon Gas Injection System (GIS) was used to deposit, in two steps, a thick layer of amorphous carbon over the area of interest. After the protective deposition, the samples were drilled and polished with a beam consisting of $\mathrm{Ga}^{+}$ions operating at an acceleration voltage of $30 \mathrm{kV}$ with subsequent current polishing steps of $2.5 \mathrm{nA}$ and $80 \mathrm{pA}$, respectively. Topography images were taken with an atomic force microscope NT-MDT Solver Nano in intermittent contact mode. Optical absorption measurements were performed with a UV-
Vis spectrometer Lambda 950 from Perkin equipped with an integrating sphere. Unless stated otherwise all average total transmittance (AVT) values are calculated by averaging the total transmittance recorded between $400 \mathrm{~nm}$ and $800 \mathrm{~nm}$. The thickness of the active layer was determined though measurements on cross-sectional micrographs and/or a profilometer, each value is then obtained as a result of averaging 10 measurements along the film. The grain size was measured with the software for interface design and characterization magnification, the average values of the grain size were then calculated by averaging 100 measurements along an area of $20 \mu \mathrm{m}^{2}$.

\section{Acknowledgements}

The authors would like to acknowledge the funding of the Erlangen Graduate School in Advanced Optical Technologies (SAOT) at the University of Erlangen-Nuremberg, which is funded by the German Research Foundation (DFG) within the framework of its "Excellence Initiative". The work was further supported by the Cluster of Excellence "Engineering of Advanced Materials" (EAM). The authors acknowledge financial support from the DFG research training group GRK 1896 at Erlangen University and from the Joint Project Helmholtz-Institute Erlangen Nürnberg (HI-ERN) under project number DBF01253, respectively. The authors would like to acknowledge the company rent a scientist (RAS) for material support. C. J. B. acknowledges the financial support through the "Aufbruch Bayern" initiative of the state of Bavaria (EnCN and solarfactory of the future), the Bavarian Invitiative "Solar Technologies go Hybrid" (SolTech) and the "Solarfactory of the future" with the Energy Campus Nürnberg (EnCN). M. S. acknowledges primary support from a fellowship by the Portuguese Fundação para a Ciência e a Tecnologia (SFRH/ BPD/71816/2010). C. O. R. Q would like to gratefully acknowledge the financial support from The Mexican National Council for Science and Technology (CONACYT). C. J. B. and K. F. would like to acknowledge the support of the EU-project SOLPROCEL ("Solution Processed High Performance Transparent Organic Photovoltaic Cells," Grant No. 604506).

\section{References}

1 Z. He, C. Zhong, S. Su, M. Xu, H. Wu and Y. Cao, Nat. Photonics, 2012, 6, 591.

2 Y. Liu, J. Zhao, Z. Li, C. Mu, W. Ma, H. Hu, K. Jiang, H. Lin, H. Ade and H. Yan, Nat. Commun., 2014, 5, 1.

3 M. Liu, M. B. Johnston and H. J. Snaith, Nature, 2013, 501, 395.

4 J. Burschka, N. Pellet, S.-J. Moon, R. Humphry-Baker, P. Gao, M. K. Nazeeruddin and M. Grätzel, Nature, 2013, 499, 316.

5 J. Xu, A. Buin, A. H. Ip, W. Li, O. Voznyy, R. Comin, M. Yuan, S. Jeon, Z. Ning, J. J. McDowell, P. Kanjanaboos, J.-P. Sun, X. Lan, L. N. Quan, D. H. Kim, I. G. Hill, P. Maksymovych and E. H. Sargent, Nat. Commun., 2015, 6, 7081.

6 N. J. Jeon, J. H. Noh, W. S. Yang, Y. C. Kim, S. Ryu, J. Seo and S. Il Seok, Nature, 2015, 517, 476.

7 W. S. Yang, J. H. Noh, N. J. Jeon, Y. C. Kim, S. Ryu, J. Seo and S. Il Seok, Science, 2015, 348, 1234. 
8 M. C. Scharber, D. Mühlbacher, M. Koppe, P. Denk, C. Waldauf, A. J. Heeger and C. J. Brabec, Adv. Mater., 2006, 18, 789.

9 T. Ameri, G. Dennler, C. Waldauf, H. Azimi, A. Seemann, K. Forberich, J. Hauch, M. Scharber, K. Hingerl and C. J. Brabec, Adv. Funct. Mater., 2010, 20, 1592.

10 R. Betancur, P. Romero-Gomez, A. Martinez-Otero, X. Elias, M. Maymó and J. Martorell, Nat. Photonics, 2013, 7, 995.

11 C. D. Bailie, M. G. Christoforo, J. P. Mailoa, A. R. Bowring, E. L. Unger, W. H. Nguyen, J. Burschka, N. Pellet, J. Z. Lee, M. Grätzel, R. Noufi, T. Buonassisi, A. Salleo and M. D. McGehee, Energy Environ. Sci., 2014, 8, 956.

12 F. Guo, H. Azimi, Y. Hou, T. Przybilla, M. Hu, C. Bronnbauer, S. Langner, E. Spiecker, K. Forberich and C. J. Brabec, Nanoscale, 2014, 7, 1642.

13 G. E. Eperon, V. M. Burlakov, A. Goriely and H. J. Snaith, ACS Nano, 2014, 8, 591.

14 P. You, Z. Liu, Q. Tai, S. Liu and F. Yan, Adv. Mater., 2015, 27, 3632.

15 Z. Li, S. A. Kulkarni, P. P. Boix, E. Shi, A. Cao, K. Fu and S. K. Batabyal, ACS Nano, 2014, 8, 6797.

16 C. Roldan, O. Malinkiewicz, R. Betancur, G. Longo, C. Momblona, F. Jaramillo, L. Camacho and H. J. Bolink, Energy Environ. Sci., 2014, 7, 2968.

17 J. Xiong, B. Yang, R. Wu, C. Cao, Y. Huang, C. Liu, Z. Hu, H. Huang, Y. Gao and J. Yang, Org. Electron., 2015, 24, 106.

18 X. Manda, H. Fuzhi, H. Wenchao, D. Yasmina, Z. Ye, E. Joanne, G.-W. Angus, B. Udo, C. Yi-Bing and S. Leone, Angew. Chem., 2014, 126, 10056.

19 A. Cannavale, G. E. Eperon, P. Cossari, A. Abate, H. Snaith and G. Gigli, Energy Environ. Sci., 2015, 8, 1578.

20 D. Liu, M. K. Gangishetty and T. L. Kelly, J. Mater. Chem. A, 2014, 2, 19873.

21 J. Xi, Z. Wu, H. Dong, B. Xia, F. Yuan, B. Jiao, L. Xiao, Q. Gong and H. Xun, Nanoscale, 2015, 7, 10699.

22 J. W. Jung, C.-C. Chueh and A. K.-Y. Jen, Adv. Energy Mater., 2015, 5, 1500486.

23 L. K. Ono, S. Wang, Y. Kato, S. R. Raga and Y. Qi, Energy Environ. Sci., 2014, 7, 3989.

24 F. Guo, N. Li, F. W. Fecher, N. Gasparini, C. O. R. Quiroz, C. Bronnbauer, Y. Hou, V. V. Radmilović, V. R. Radmilović, E. Spiecker, K. Forberich and C. J. Brabec, Nat. Commun., 2015, 6, 7730.

25 C. J. M. Emmott, A. Urbina and J. Nelson, Sol. Energy Mater. Sol. Cells, 2012, 97, 14.

26 Y. Shao, Z. Xiao, C. Bi, Y. Yuan and J. Huang, Nat. Commun., 2014, 5, 5784 .
27 Y. Hou, H. Zhang, W. Chen, S. Chen, C. O. R. Quiroz, H. Azimi, A. Osvet, G. J. Matt, E. Zeira, J. Seuring, N. Kausch-Busies, W. Lövenich and C. J. Brabec, Adv. Energy Mater., 2015, 5, 1500543.

28 N. J. Jeon, J. H. Noh, Y. C. Kim, W. S. Yang, S. Ryu and S. Il Seok, Nat. Mater., 2014, 13, 897.

29 M. Zhang, H. Yu, J.-H. Yun, M. Lyu, Q. Wang and L. Wang, Chem. Commun., 2015, 51, 10038.

30 K. Hwang, Y. Jung, Y. Heo, F. H. Scholes, S. E. Watkins, J. Subbiah, D. J. Jones, D. Kim and D. Vak, Adv. Mater., 2015, 1241.

31 Q. Lin, A. Armin, R. Chandra, R. Nagiri, P. L. Burn and P. Meredith, Nat. Photonics, 2015, 9, 106.

32 S. Colella, E. Mosconi, P. Fedeli, A. Listorti, F. Gazza, F. Orlandi, P. Ferro, T. Besagni, A. Rizzo, G. Calestani, G. Gigli, F. de Angelis and R. Mosca, Chem. Mater., 2013, 25, 4613-4618.

33 M. M. Lee, J. Teuscher, T. Miyasaka, T. N. Murakami and H. J. Snaith, Science, 2012, 338, 643.

34 W. Nie, H. Tsai, R. Asadpour, A. J. Neukirch, G. Gupta, J. J. Crochet, M. Chhowalla, S. Tretiak, M. A. Alam and H. Wang, Science, 2015, 347, 522.

35 F. Verbakel, S. C. J. Meskers and R. A. J. Janssen, Appl. Phys. Lett., 2006, 89, 102103.

36 A. Armin, M. Velusamy, P. Wolfer, Y. Zhang, P. L. Burn and P. Meredith, ACS Photonics, 2014, 1, 173-181.

37 G. F. Burkhard, E. T. Hoke and M. D. McGehee, Adv. Mater., 2010, 22, 3293.

38 B. Yang, O. Dyck, J. Poplawsky, J. Keum, A. Puretzky, S. Das, I. N. Ivanov, C. M. Rouleau, G. Duscher, D. B. Geohegan and K. Xiao, J. Am. Chem. Soc., 2015, 137, 9210-9213.

39 M. I. Saidaminov, A. L. Abdelhady, B. Murali, E. Alarousu, V. M. Burlakov, W. Peng, I. Dursun, L. Wang, Y. He, G. Maculan, A. Goriely, T. Wu, O. F. Mohammed and O. M. Bakr, Nat. Commun., 2015, 6, 7586.

40 D. Shi, V. Adinolfi, R. Comin, M. Yuan, E. Alarousu, A. Buin, Y. Chen, S. Hoogland, A. Rothenberger, K. Katsiev, Y. Losovyj, X. Zhang, P. A. Dowben, O. F. Mohammed, E. H. Sargent and O. M. Bakr, Science, 2015, 347, 519.

41 Y. Wu, A. Islam, X. Yang, C. Qin, J. Liu, K. Zhang, W. Peng and L. Han, Energy Environ. Sci., 2014, 7, 2934.

42 N. Ahn, D.-Y. Son, I.-H. Jang, S. M. Kang, M. Choi and N.-G. Park, J. Am. Chem. Soc., 2015, 137, 8696-8699.

43 P. Nanocrystals, F. Zhu, L. Men, Y. Guo, Q. Zhu, U. Bhattacharjee and P. M. Goodwin, ACS Nano, 2015, 2948. 44 Y. Zhou, M. Yang, W. Wu, A. L. Vasiliev, K. Zhu and N. P. Padture, J. Mater. Chem. A, 2015, 3, 8178. 